\title{
What do we learn from a Genome Wide Association Study performed on HIV-I infected Long Term Non Progressors individuals?
}

\author{
Julien Guergnon*1, Cyril Dalmasso ${ }^{2}$, Ioannis Theodorou ${ }^{1}$, Agostino Riva ${ }^{3}$ for \\ the GISHEAL and PRIMO studies groups ${ }^{4,5}$
}

\begin{abstract}
Address: ${ }^{1}$ INSERM UMR-S 945, School of Medicine Pierre et Marie Curie, Paris, France, ${ }^{2}$ University Paris 11, Villejuif, France and Genome Institute of Singapore, Singapore, ${ }^{3}$ Department of Cytomorphology, School of Medicine, University of Cagliar, Cagliari, Italy, ${ }^{4} \mathrm{GISHEAL}$ study group School of Medicine Pierre et Marie Curie, Paris, France; Dominique Costagliola, INSERM U720, University Pierre et Marie Curie, Paris, France and ${ }_{5}^{5}$ PRIMO study group INSERM U802, University Paris-Sud 11, Le Kremlin Bicêtre, France

* Corresponding author
\end{abstract}

from Frontiers of Retrovirology: Complex retroviruses, retroelements and their hosts

Montpellier, France. 21-23 September 2009

Published: 24 September 2009

Retrovirology 2009, 6(Suppl 2):O27 doi:10.1 I86/I742-4690-6-S2-O27

This abstract is available from: http://www.retrovirology.com/content/6/S2/O27

(C) 2009 Guergnon et al; licensee BioMed Central Ltd.

\section{Background}

Previous Genome Wide Association Studies performed on Elite Controllers and control HIV-1 infected individuals have shown that the MHC locus is predominantly responsible for containing plasma viremia below a threshold of detection. Here we performed a GWAS on a cohort of 160 HIV-1 infected Caucasian Long Term Non Progressors (LTNP) from the EC-funded European-African "GISHEAL" Consortium in order to explore whether novel genetic factors could account for the LTNP phenotype (i.e. maintenance of CD4 T cell counts $>500$ cells $/ \mu \mathrm{l}$ and good health conditions without therapy).

\section{Results}

Frequencies of the SNPs found in LTNP were challenged vs. those of seroconverters of the French "PRIMO" cohort. Most of the SNPs strongly associated to LTNP phenotype were found in the MHC region (figure 1), especially encompassing class I and class III genes. Since 6 of the 10 top SNPs are in the HLA-B region, we confirm previous studies showing that class I HLA-B27 and HLA-B57 alleles are strongly correlated to the LTNP condition. In addition, about $65 \%$ of our LTNP naturally resist to HIV disease progression independently of HLA-B27/B57; in this regard, quite strikingly, 11 over 32 SNPs with a Q-value <
0.05 are located in MHC class III region in GISHEAL LTNP cohort.

\section{Conclusion}

Thus, our findings support the concept that different MHC loci significantly contribute to long-term control of HIV disease progression in the absence of antiretroviral therapy and provide novel evidence of a seminal role of MHC class III gene polymorphisms in determining the LTNP phenotype. 